\title{
Speed tracking control for an uncertain permanent magnet synchronous motor drive system
}

\author{
Tat-Bao-Thien Nguyen ${ }^{\mathrm{a}}$, Teh-Lu Liao ${ }^{\mathrm{a}}$, Hang-Hong Kuo ${ }^{\mathrm{a}}$, Jun-Juh Yan ${ }^{\mathrm{b}, *}$ \\ ${ }^{a}$ Department of Engineering Science, National Cheng Kung University, Tainan 701, Taiwan \\ ${ }^{\mathrm{b}}$ Department of Computer and Communication, Shu-Te University, Kaohsiung 824, Taiwan \\ *Corresponding Author: jjyan@ stu.edu.tw
}

\begin{abstract}
In this paper, a new adaptive controller is developed to suppress chaos and gain advanced speed tracking in a permanent magnet synchronous motor (PMSM) with unknown parameters and uncertainties. The controller has two parts: fuzzy neural and compensatory controllers. The fuzzy controller estimates the ideal feedback control law, while the compensatory controller is used to reduce the effects of the estimation error. With the improved controller design, the controller not only meets the control objective but also surely avoids the singularity problem that usually appears in indirect adaptive control techniques based on fuzzy/neural networks estimation. Finally, numerical simulations are executed to verify the validity of the proposed method.
\end{abstract}

Keywords: adaptive control, fuzzy control, neural networks, permanent magnet synchronous motor.

\section{Introduction}

Chaos is a special phenomenon of nonlinear dynamical systems in that the system dynamics is highly sensitive to initial conditions [1]. Consequently, the small differences in initial conditions yield widely diverging outcomes for such dynamical systems. Except sensitiveness to initial conditions, irregular, unpredictable behavior and aperiodic orbits are also properties of chaotic systems. The first study of the chaotic phenomenon in permanent magnet synchronous motors (PMSMs) is given by $\mathrm{Li}$, et al. [2]. Their results indicated that a PMSM can fall into chaos when its parameters lie in a certain area. Since chaotic oscillations can degrade the stabilization or even cause the system to collapse, eliminating and controlling chaos in PMSMs have received much attention from many scholars. So far, various control methods have been developed for chaotic PMSMs, including feedback linearization, sliding mode control, quasi-sliding mode control, and so on [3-8]. However, they still have shortcomings. Most of them require the exact mathematical models to calculate the control laws, that is, they may not be used when the mathematical models are dynamic due to undesired uncertainties.

Nowadays, fuzzy logic and neural networks (NN) have been widely applied to control engineering. They are considered as powerful tools for modeling and controlling highly uncertain, nonlinear and complex systems [9-13]. Numerous researchers have developed fuzzy systems and neural networks, and applied them to PMSMs successfully [14-18]. Although these control techniques can exhibit the good performance in PMSMs, some weaknesses remain. The fuzzy guaranteed cost control method [14] may become problematic when the desired point is not at the origin. In self organizing fuzzy sliding mode control [15], the values of fuzzy gain scaling factors are not easy to find for a particular PMSM. For the adaptive fuzzy control method in [16], the controller may get the awful performance if the reference point is not a constant. In addition, the tracking error just converges to a small neighborhood of the origin instead of the origin. Although the indirect adaptive control method in [18] showed the advanced tracking performance, it may face to the singularity problem when the neural network operates in the initial period.

Because the existing control methods mentioned above still have weaknesses and inspired from the potential 
applications of fuzzy neural networks, we develop a new adaptive controller for a chaotic PMSM to control chaos and ensure the perfect tracking performance in that the tracking error can converge to the origin. The controller uses a fuzzy neural network to estimate the unknown nonlinear terms and construct the nonlinear control law. The effect of estimation errors is also considered and treated by an additional controller. Moreover, the new design of developed controller allows it to avoid the singularity problem that usually appears in indirect adaptive control techniques based on fuzzy/neural networks estimation.

This paper is organized as follows. The dynamics of a chaotic PMSM and the formulation of control problem are described in Section 2. The design of the controller is expressed in Section 3. In Section 4, simulation results are illustrated. Finally, the conclusion is offered in Section 5.

\section{Dynamic model of PMSM and problem formulation}

The dynamic model of a PMSM with the smooth air gap can be expressed by the following equations [2]:

$$
\begin{aligned}
& \dot{x}_{1}=\sigma\left(x_{3}-x_{1}\right)+\tilde{T}_{L}, \\
& \dot{x}_{2}=-x_{2}-x_{3} x_{1}+x_{1}+\tilde{u}_{q}, \\
& \dot{x}_{3}=-x_{2}+x_{3} x_{1}+\tilde{u}_{d},
\end{aligned}
$$

where $x_{1}, x_{2}$ and $x_{3}$ are state variables, which denote angle speed, quadrature and direct axis currents respectively. $\sigma$ and $\gamma$ are system parameters. $\tilde{T}_{L}, \tilde{u}_{d}$ and $\tilde{u}_{q}$ express the load torque, direct and quadrature axis voltages respectively.

When the external inputs are set to zero, that is, $\tilde{T}_{L}=\tilde{u}_{d}=\tilde{u}_{q}=0$, the system in (1) becomes an unforced system as [2]

$$
\begin{aligned}
& \dot{x}_{1}=\sigma\left(x_{3}-x_{1}\right), \\
& \dot{x}_{2}=-x_{2}-x_{3} x_{1}+2 x_{1}, \\
& \dot{x}_{3}=-x_{2}+x_{3} x_{1} .
\end{aligned}
$$

The system in (2) falls into chaos when the system parameters are set as $\sigma=5.45$ and $\gamma=20$ [2]. Fig. 1 shows the typical chaotic attractor of a PMSM with initial states $\left[\begin{array}{lll}x_{1}(0) & x_{2}(0) & x_{3}(0)\end{array}\right]^{T}=\left[\begin{array}{lll}1 & -1 & 0\end{array}\right]^{T}$.

In order to control the system in (2), we add control input $u$ and we suppose that the system experiences the bounded uncertainties $d_{i}(x, t) \in R, i=1,2,3$. Then the system with control input $u$ can be expressed as:

$$
\begin{aligned}
& \dot{x}_{1}=\sigma\left(x_{2}-x_{1}\right)+d_{1}, \\
& \dot{x}_{2}=-x_{2}-x_{1} x_{3}+x_{1}+d_{2}+u, \\
& \dot{x}_{3}=-x_{3}+x_{2} x_{1}+d_{3},
\end{aligned}
$$

The control objective is to design a controller that can suppress chaos and allow the output $y(t)=x_{1}$ to track the desired trajectory $y_{d}(t) \in R$ which is supposed to be smooth up to second order. Then, the controlled system in (3) with output $y(t)=x_{1}$ can be expressed in SISO form as follows:

$$
\left\{\begin{array}{l}
\dot{x}=f(x)+g(x) u \\
y=h(x)
\end{array}\right.
$$

where $x=\left[\begin{array}{l}x_{1} \\ x_{2} \\ x_{3}\end{array}\right], f(x)=\left[\begin{array}{c}\sigma\left(x_{2}-x_{1}\right)+d_{1} \\ -x_{2}-x_{1} x_{3}+x_{1}+d_{2} \\ -x_{3}+x_{2} x_{1}+d_{3}\end{array}\right]$,

$$
g(x)=\left[\begin{array}{l}
0 \\
1 \\
0
\end{array}\right] \text { and } h(x)=x_{1} .
$$

Since the system in (4) has relative degree $r=2$, the change of coordinates

$$
\begin{aligned}
& z_{1}=y, \\
& z_{2}=L_{f} h(x)=\sigma\left(x_{2}-x_{1}\right)+d_{1},
\end{aligned}
$$

will lead to

$$
\begin{aligned}
& \dot{z}_{1}=z_{2}, \\
& \dot{z}_{2}=a(x)+b(x) u, \\
& y=z_{1},
\end{aligned}
$$

where

$$
\begin{aligned}
& a(x)=L_{f}^{2} h(x) \\
& =\left(-\sigma+\frac{\partial d_{1}}{\partial x_{1}}\right)\left(\sigma x_{2}-\sigma x_{1}+d_{1}\right) \\
& +\left(\sigma+\frac{\partial d_{1}}{\partial x_{2}}\right)\left(-x_{2}-x_{1} x_{3}+x_{1}+d_{2}\right) \\
& +\frac{\partial d_{1}}{\partial x_{3}}\left(-x_{3}+x_{1} x_{3}+d_{3}\right),
\end{aligned}
$$




$$
b(x)=L_{g} L_{f} h(x)=\sigma+\frac{\partial d_{1}}{\partial x_{2}}
$$

In order to meet the control objective, based on the feedback linearization, the ideal nonlinear controller $u^{*}(x)$ can be chosen as:

$$
u^{*}(x)=\frac{1}{b(x)}(-a(x)+v(t))
$$

where $v(t) \in R$ is the new variable and it can be calculated as

$$
v(t)=\ddot{y}_{d}(t)+\bar{e}_{s}(t)+\eta e_{s}(t),
$$

where $\eta$ is a positive factor, and $e_{s}(t)$ and $\bar{e}_{s}(t)$ can be calculated by

$$
\begin{aligned}
& e_{s}(t)=\dot{e}_{0}(t)+k e_{0}(t), \\
& \bar{e}_{s}(t)=\dot{e}_{s}(t)-\ddot{e}_{0}(t)=k \dot{e}_{0}(t),
\end{aligned}
$$

where $e_{0}(t)=y_{d}(t)-y(t)$ is tracking error, and $k$ is chosen to ensure that $\Delta(s)=s+k$ is a Hurwitz polynomial.

In order to ensure (9) proper, we suppose that $b(x)$ is lower bounded by a known positive constant $\underline{b}$, that is, $0<\underline{b} \leq b(x)<\infty$.

Moreover, the zero dynamics is also considered. Equation $\quad \dot{z}_{3}=\dot{x}_{3}=-z_{3}+\frac{1}{\sigma} z_{1}\left(z_{2}+\sigma z_{1}-d_{1}\right)+d_{3}$ describes the additional state equation and $\dot{z}_{3}=-z_{3}+d_{3}$ expresses the stable zero dynamics.

However, there is a problem can be arisen. Since $\sigma$, $\gamma$ and $d_{i}, i=1,2,3$, are unknown, $a(x)$ and $b(x)$ cannot be determined exactly, leading to deep difficulties on performance of the ideal control law in (9). In order to overcome this problem, we use fuzzy neural networks to estimate $a(x)$ and $b(x)$.

\section{Adaptive controller based on fuzzy neural networks}

Let $\hat{a}(x, t)$ and $\hat{b}(x, t)$ be the estimations of $a(x)$ and $b(x)$ respectively. Then, $\hat{a}(x, t)$ and $\hat{b}(x, t)$ are calculated by a fuzzy neural network according to the following equations:

$$
\hat{a}(x, t)=W_{a}^{T}(t) S(x),
$$

$$
\hat{b}(x, t)=W_{b}^{T}(t) S(x)
$$

where

$$
W_{a}(t)=\left[\begin{array}{llll}
w_{a 1} & w_{a 2} & \ldots & w_{a 9}
\end{array}\right]
$$

$W_{b}(t)=\left[\begin{array}{llll}w_{b 1} & w_{b 2} & \ldots & w_{b 9}\end{array}\right]$ are weighting vectors described as the links between rule layer and output layer in Fig. 2, while $S(x)=\left[\begin{array}{llll}s_{1}(x) & s_{2}(x) & \ldots & s_{9}(x)\end{array}\right]$ is the fuzzy basic vector of which each element $s_{i}(x), \quad i=1,2 \ldots 9$ is defined by

$$
s_{i}(x)=\frac{\prod_{j=1}^{3} \mu_{A_{j}^{i}}(x)}{\sum_{i=1}^{9}\left(\prod_{j=1}^{3} \mu_{A_{j}^{i}}(x)\right)},
$$

where $\mu_{A_{j}^{i}}, i, j=1,2 . .9$, is a membership function.

During the operation time, $W_{a}(t)$ and $W_{b}(t)$ are online tuned so that $\hat{a}(x, t)$ and $\hat{b}(x, t)$ converge to $a(x)$ and $b(x)$ respectively. Here we chose adaptive laws for $W_{a}(t)$ and $W_{b}(t)$ as

$$
\begin{aligned}
& \dot{W}_{a}(t)=-Q_{a}^{-1} S(x) e_{s}(t), \\
& \dot{W}_{b}(t)=-Q_{b}^{-1} S(x) u_{n n}(x) e_{s}(t),
\end{aligned}
$$

where $Q_{a}$ and $Q_{b}$ are positive-definite weighting matrices.

Using the estimations, $\hat{a}(x, t)$ and $\hat{b}(x, t)$, and 
following the certainty equivalent approach, the fuzzy neural controller $u_{n n}(x)$ based on the ideal control law (9) can be obtained as

$$
u_{n n}(x)=\frac{1}{\hat{b}(x, t)}(-\hat{a}(x, t)+v(t)) .
$$

However, the control law in (6) may fall into the singularity problem when $\hat{b}(x, t)$ tends to zero or even receives the zero value in some point in time initially. In order to avoid this problem, we replace the control law in (18) with

$$
u_{n n}(x)=\frac{\hat{b}(x, t)}{\hat{b}^{2}(x, t)+\varepsilon}(-\hat{a}(x, t)+v(t)),
$$

where $\varepsilon$ is a nonzero constant which guarantees that the term $\hat{b}^{2}(x, t)+\varepsilon$ is always nonzero.

Since a fuzzy neural network is used to estimate $a(x)$ and $b(x)$, estimation errors always exist. In this study, we suppose that the estimation errors are bounded by known constants, $\bar{\delta}_{a}>0$ and $\bar{\delta}_{b}>0$, respectively. Then to reduce the effect of estimation errors to the controlled system, we add a compensatory controller $u_{c}(x)$ described by

$$
u_{c}(x)=\frac{1}{\underline{b}}\left(\bar{\delta}_{a}+\bar{\delta}_{b}\left|u_{n n}(x)\right|+\left|u_{r}(x)\right|\right) \operatorname{sgn}\left(e_{s}(t)\right),
$$

where $u_{r}(x)=\frac{\varepsilon}{\hat{b}^{2}(x, t)+\varepsilon}(-\hat{a}(x, t)+v(t))$.

Therefore, the total controller $u(x)$ is the sum of the fuzzy neural controller $u_{n n}(x)$ and the compensatory controller $u_{c}(x)$ as

$$
\begin{aligned}
u(x)= & u_{n n}(x)+u_{c}(x) \\
= & u_{n n}(x) \\
& +\frac{1}{b}\left(\bar{\delta}_{a}+\bar{\delta}_{b}\left|u_{n n}(x)\right|+\left|u_{r}(x)\right|\right) \operatorname{sgn}\left(e_{s}(t)\right) \\
= & \frac{\hat{b}(x, t)}{\hat{b}^{2}(x, t)+\varepsilon}(-\hat{a}(x, t)+v(t)) \\
& +\frac{1}{\underline{b}}\left(\bar{\delta}_{a}+\bar{\delta}_{b}\left|u_{n n}(x)\right|+\left|u_{r}(x)\right|\right) \operatorname{sgn}\left(e_{s}(t)\right)
\end{aligned}
$$

Finally, for stability analysis, Lyapunov approach can be used to ensure the stability of the controlled system.

\section{Simulation study}

In this section, numerical simulations are carried out to verify the validity of the proposed method. Firstly, we consider the system without control action and uncertainties. The simulation results show the chaotic oscillations of the state response, as depicted in Fig. 3.

Secondly, we use the proposed controller to suppress chaos and track the desired speed in the PMSM under the effect of uncertainties. The bounded uncertainties were chosen as $d_{1}=1+\cos (t), \quad d_{2}=-1$ and $d_{3}=\sin \left(x_{1}\right)$ for simulation, while the chosen desired trajectory is $y_{d}(t)=\sin (\pi t)$. Then, the control parameters are chosen as follows:

$$
\begin{aligned}
& k=40, \eta=60, \underline{b}=1, \quad \varepsilon=1, \bar{\delta}_{a}=\bar{\delta}_{b}=0.1, \\
& Q_{a}=40 * \operatorname{eye}(9) \text { and } Q_{b}=20 * \operatorname{eye}(9) .
\end{aligned}
$$

Since the input states of the controller are normalized in a range of $[-1,1]$, nine membership functions are chosen as

$$
\begin{array}{ll}
\mu_{A_{j}^{1}}(x)=\exp \left[-\frac{(x+1)^{2}}{2 * 0.2^{2}}\right], & \mu_{A_{j}^{2}}(x)=\exp \left[-\frac{(x+0.75)^{2}}{2 * 0.2^{2}}\right], \\
\mu_{A_{j}^{3}}(x)=\exp \left[-\frac{(x+0.5)^{2}}{2 * 0.2^{2}}\right], & \mu_{A_{j}^{4}}(x)=\exp \left[-\frac{(x+0.25)^{2}}{2 * 0.2^{2}}\right],
\end{array}
$$

$\mu_{A_{j}^{5}}(x)=\exp \left[-\frac{(x+0)^{2}}{2 * 0.2^{2}}\right]$

$$
\mu_{A_{j}^{6}}(x)=\exp \left[-\frac{(x-0.25)^{2}}{2 * 0.2^{2}}\right]
$$

$\mu_{A_{j}^{7}}(x)=\exp \left[-\frac{(x-0.5)^{2}}{2 * 0.2^{2}}\right]$,

$$
\mu_{A_{j}^{8}}(x)=\exp \left[-\frac{(x-0.75)^{2}}{2 * 0.2^{2}}\right]
$$

and $\mu_{A_{j}^{9}}(x)=\exp \left[-\frac{(x-1)^{2}}{2 * 0.2^{2}}\right]$

The results, as shown in Fig. 4,5 illustrate that the chaotic oscillations are completely removed and the speed of a PMSM follows the desired trajectory perfectly.

\section{Conclusions}


In this paper, an improved adaptive controller has been developed to control chaotic PMSM successfully. Moreover, the new design of controller can avoid the singularity problem and ensure perfect tracking performance in that the tracking error converges to zero asymptotically even with initial phase. Simulation results are provided to illustrate the advanced functions of proposed method.

\section{Acknowledgment}

The authors gratefully acknowledge the support of National Science Council of Taiwan through the grants NSC101-2221-E-006-190-MY2 and NSC102-2221-E-366003.

\section{References}

[1] S. H. Strogatz: Nonlinear dynamics and chaos: with applications to physics, biology and chemistry: Perseus publishing, 2001.

[2] Z. Li, J. B. Park, Y. H. Joo, B. Zhang, and G. Chen: "Bifurcations and chaos in a permanent-magnet synchronous motor," Circuits and Systems I: Fundamental Theory and Applications, IEEE Transactions on, vol. 49, pp. 383-387, 2002.

[3] Y. Gao and K. T. Chau: "Chaotification of permanent-magnet synchronous motor drives using time-delay feedback," in IECON 02 [Industrial Electronics Society, IEEE 2002 28th Annual Conference of the], Sevilla, Spain, 2002, pp. 762-766 vol.1.

[4] X. Ge and J. Huang: "Chaos control of permanent magnet synchronous motor," in Electrical Machines and Systems, 2005. ICEMS 2005. Proceedings of the Eighth International Conference on, Nanjing, China, 2005, pp. 484-488 Vol. 1.

[5] R. Haipeng and L. Ding: "Nonlinear feedback control of chaos in permanent magnet synchronous motor," Circuits and Systems II: Express Briefs, IEEE Transactions on, vol. 53, pp. 45-50, 2006.

[6] C. Ma, L. Wang, Z. Yin, J. Liu, and D. Chen: "Sliding mode control of chaos in the noise-perturbed permanent magnet synchronous motor with non-smooth air-gap," Mining Science and Technology (China), vol. 21, pp. 835-838,
2011.

[7] C.-F. Huang, J.-S. Lin, T.-L. Liao, C.-Y. Chen, and J.-J. Yan: "Quasi-Sliding Mode Control of Chaos in Permanent Magnet Synchronous Motor," Mathematical Problems in Engineering, vol. 2011, 2011.

[8] C.-F. Huang, T.-L. Liao, C.-Y. Chen, and J.-J. Yan: "The design of quasi-sliding mode control for a permanent magnet synchronous motor with unmatched uncertainties," Computers \& Mathematics with Applications, vol. 64, pp. 1036-1043, 2012.

[9] F. o. Beaufays, Y. Abdel-Magid, and B. Widrow: "Application of neural networks to load-frequency control in power systems," Neural Networks, vol. 7, pp. 183-194, 1994.

[10] X. P. Cheng and R. V. Patel: "Neural network based tracking control of a flexible macro-micro manipulator system," Neural Netw., vol. 16, pp. 271-286, 2003.

[11] N. G. Chalhoub and B. A. Bazzi: "Fuzzy Logic Control for an Integrated System of a Micro-Manipulator with a Single Flexible Beam," Journal of Vibration and Control, vol. 10, pp. 755-776, 2004.

[12] H.-W. Ge, F. Qian, Y.-C. Liang, W.-1. Du, and L. Wang: "Identification and control of nonlinear systems by a dissimilation particle swarm optimization-based Elman neural network," Nonlinear Analysis: Real World Applications, vol. 9, pp. 1345-1360, 2008.

[13] W. Qin, Y. Yang, and J. Zhang: "Controlling the chaotic response to a prospective external signal using back-propagation neural networks," Nonlinear Analysis: Real World Applications, vol. 10, pp. 2985-2989, 2009.

[14] Y. Shen, Y. Zhou, and Z. Ji: "Fuzzy Guaranteed Cost Control of Permanent Magnet Synchronous Motor Chaos Systems via Fuzzy Lyapunov Function Approach," in Industrial Electronics and Applications, 2007. ICIEA 2007. 2nd IEEE Conference on, 2007, pp. 2449-2454.

[15] Y. Guo and H. Long: "Self organizing fuzzy sliding mode controller for the position control of a permanent magnet synchronous motor drive," Ain Shams Engineering Journal, vol. 2, pp. 109-118, 2011.

[16] J. Yu, B. Chen, H. Yu, and J. Gao: "Adaptive fuzzy 
tracking control for the chaotic permanent magnet synchronous motor drive system via backstepping," Nonlinear Analysis: Real World Applications, vol. 12, pp. 671-681, 2011.

[17] T.-B.-T. Nguyen, T.-L. Liao, and J.-J. Yan: "Adaptive tracking control for an uncertain chaotic permanent magnet synchronous motor based on fuzzy neural networks," Journal of Vibration and Control, July 8, 2013.

[18] T.-B.-T. Nguyen, T.-L. Liao, and J.-J. Yan: "Adaptive Sliding Mode Control of Chaos in Permanent Magnet Synchronous Motor via Fuzzy Neural Networks," Mathematical Problems in Engineering, vol. 2014, p. 11, 2014.
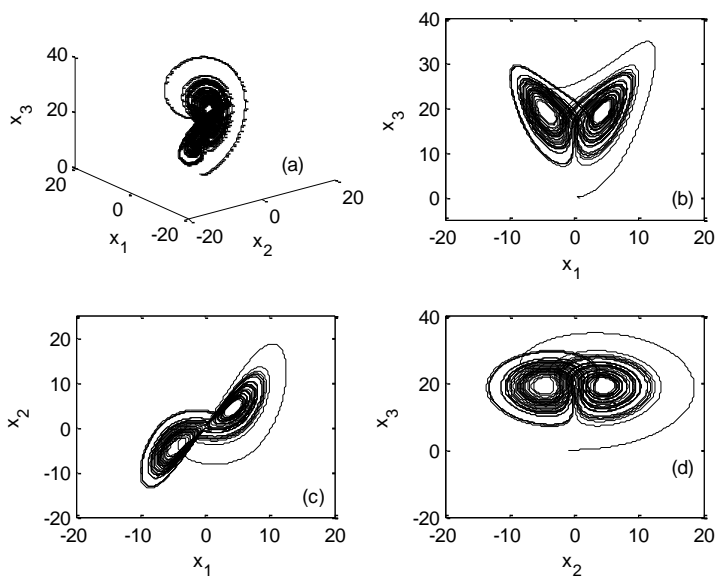

Fig. 1. (a) Typical chaotic attractor in a PMSM. (b) Chaotic motion on $x_{1}-x_{3}$ plane. (c) Chaotic motion on $x_{1}-x_{2}$ plane. (d) Chaotic motion on $x_{2}-x_{3}$ plane.

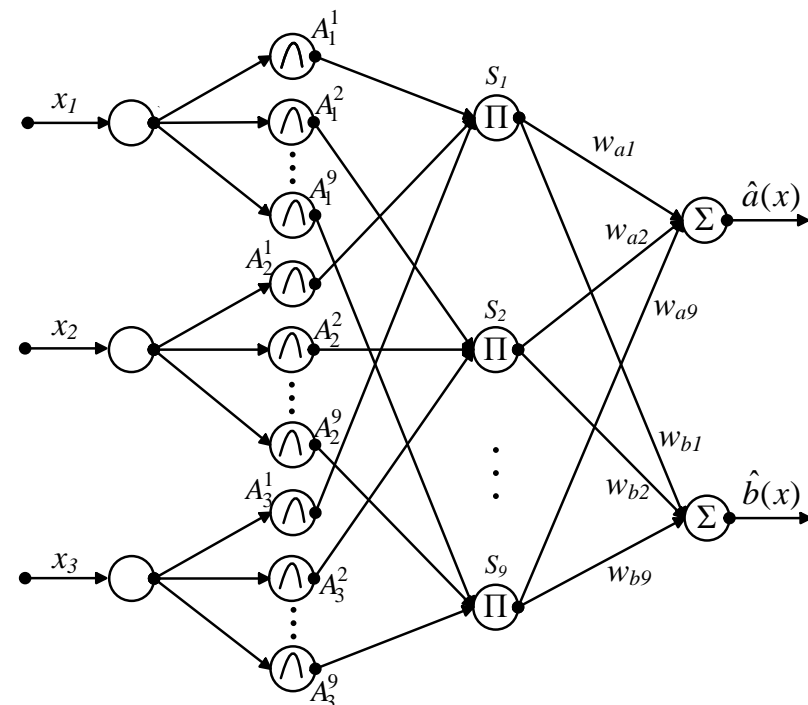

Input layer Membership layer Rule layer Output layer

Fig. 2. Structure of a fuzzy neural network.
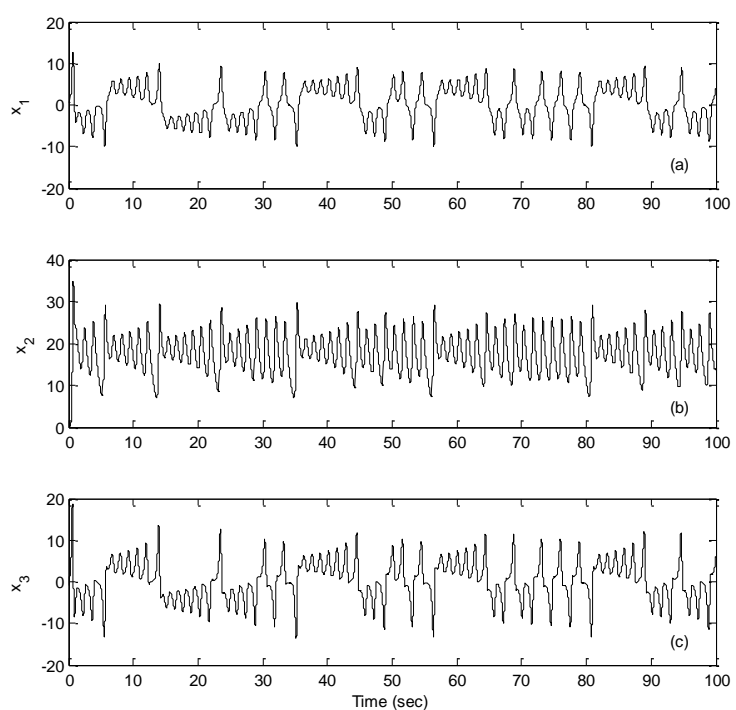

Fig. 3. Chaotic oscillations of a PMSM. 

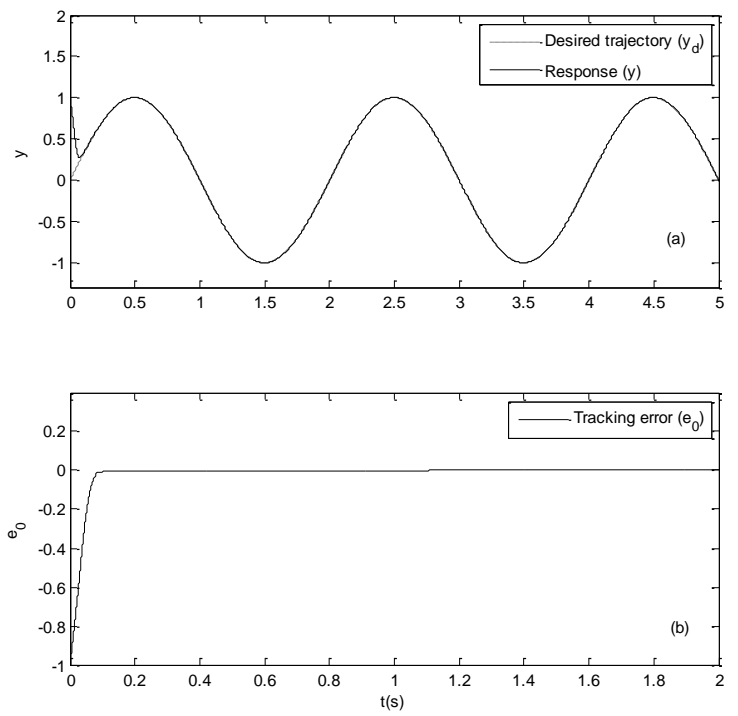

Fig. 4. Chaos suppression and speed tracking of the controlled PMSM.
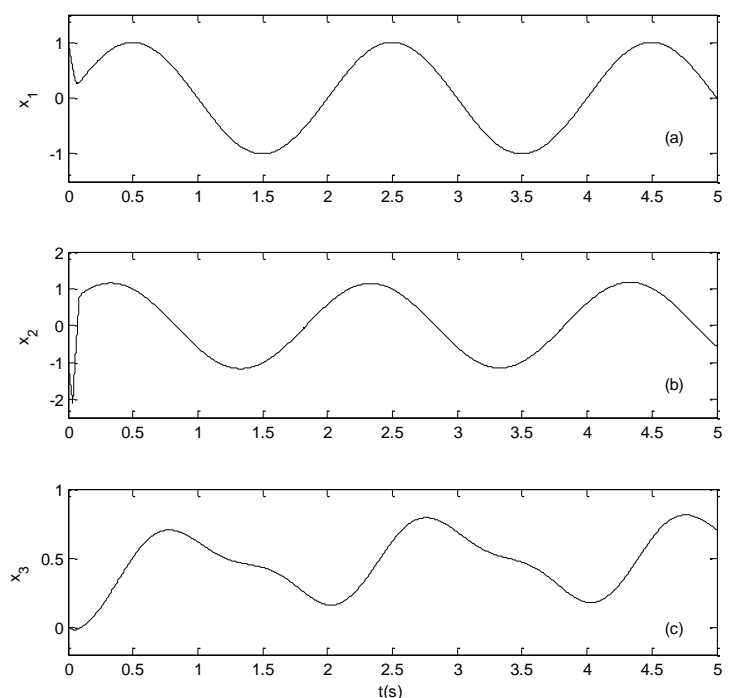

Fig. 5. State response of the controlled PMSM in $x$ coordinate. 